\title{
Selenium concentration in Korean patients with thyroid disease: A preliminary report.
}

Young Nam Kim, Joo Young Jang, Hye-In Kim, Hosu Kim, Tae Hyuk Kim, Sun Wook Kim, Jae Hoon Chung

Division of Endocrinology and Metabolism, Department of Medicine, Samsung Medical Center,

Sungkyunkwan University School of Medicine, Seoul, Republic of Korea

\section{BACKGROUND}

Selenium is an important trace element for thyroid hormone metabolism and its deficiency can cause hypothyroidism. Serum or plasma selenium concentration has been known as best biomarker which reflects selenium intake and reserve.

\section{OBJECTIVE}

We preliminarily assessed the serum or urine selenium concentrations in patients with thyroid disease compared to healthy normal population. We also investigated the correlation of serum selenium concentration with urine selenium concentration, thyroid hormone levels as well as urinary iodine concentration (UIC).

\section{METHODS}

A total of 97 patients ( 32 men, 65 women, $52.4 \pm 14.7$ years) with benign thyroid nodule or thyroid dysfunction who visited the Thyroid Center at Samsung Medical Center between 2008 and 2013 were included. Data of 175 healthy subjects provided by Lee et al. were used as control. Serum T3, free T4, and TSH concentrations were measured using commercialized RIA or IRMA kits. Serum/urine selenium and UIC were measured by inductively coupled plasma-mass spectrometry (ICPMS).

\section{RESULTS}

Comparison of serum and urine selenium concentrations of patients with thyroid disease to those of healthy subjects

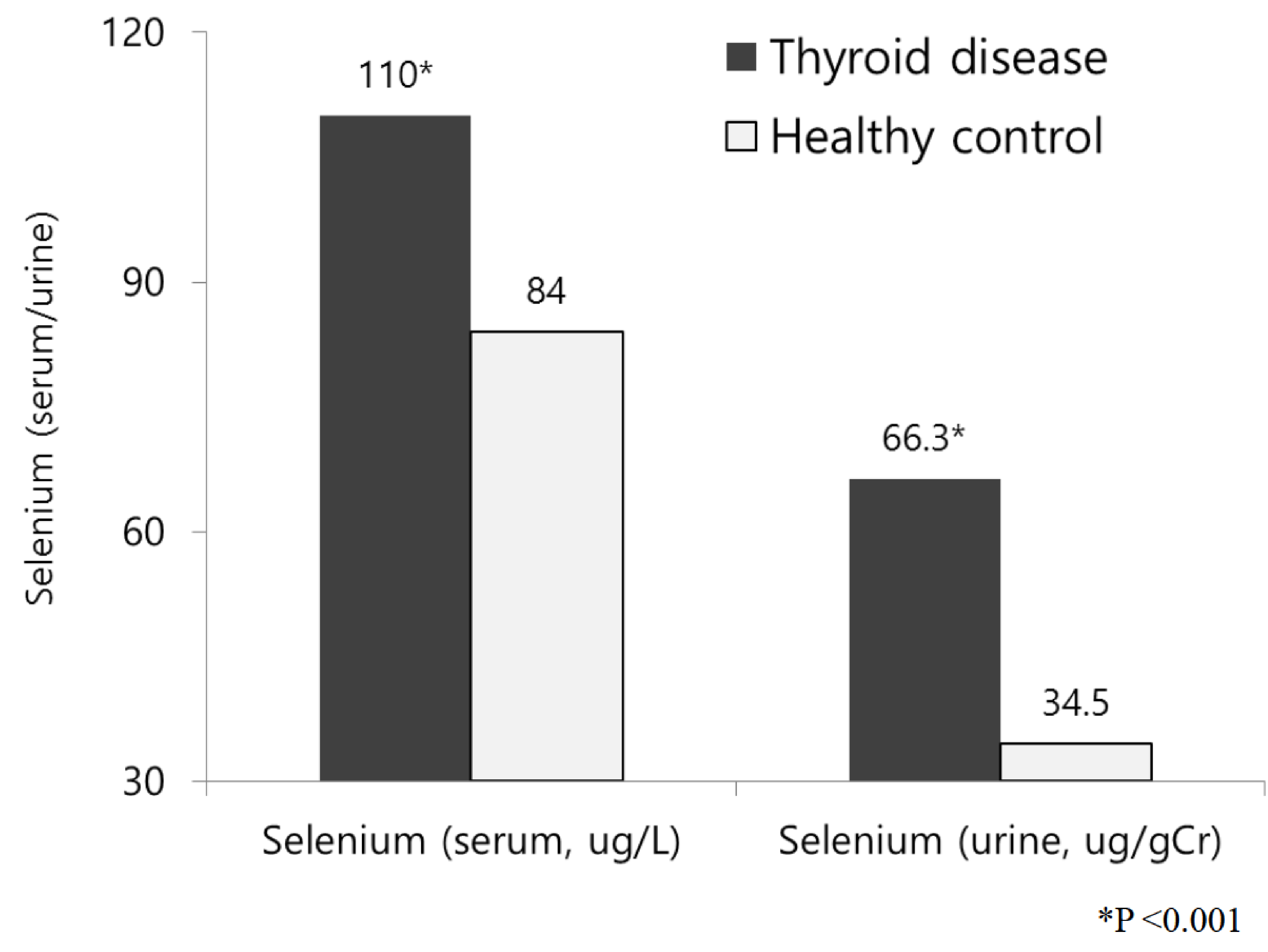

Median serum selenium concentration of the 97 patients with thyroid disease was $110 \mu \mathrm{g} / \mathrm{L}(95 \% \mathrm{CI}, 73-156)$ with a range from 67 to 169 $\mu \mathrm{g} / \mathrm{L}$. When compared to 103 healthy subjects (median $84 \mu \mathrm{g} / \mathrm{L}, 95 \% \mathrm{CI}$ $30-144)$, serum selenium concentrations of the patients with thyroid disease were significantly higher than those of healthy subjects $(\mathrm{p}<0.001)$. Median urine selenium concentration of the 69 patients with thyroid disease was $66.3 \mu \mathrm{g} / \mathrm{gCr}(95 \% \mathrm{CI}, 28.7-283.5)$ with a range from 14.4 to $489.0 \mu \mathrm{g} / \mathrm{gCr}$. When compared to 175 healthy subjects (median $34.5 \mu \mathrm{g} / \mathrm{gCr}, 95 \%$ CI 0.8-107.2), urine selenium concentrations of the patients with thyroid disease were also significantly higher than those of healthy subjects $(\mathrm{p}<0.001)$.

\section{Comparison of serum selenium concentrations of patients with various thyroid diseases to those of healthy subjects}

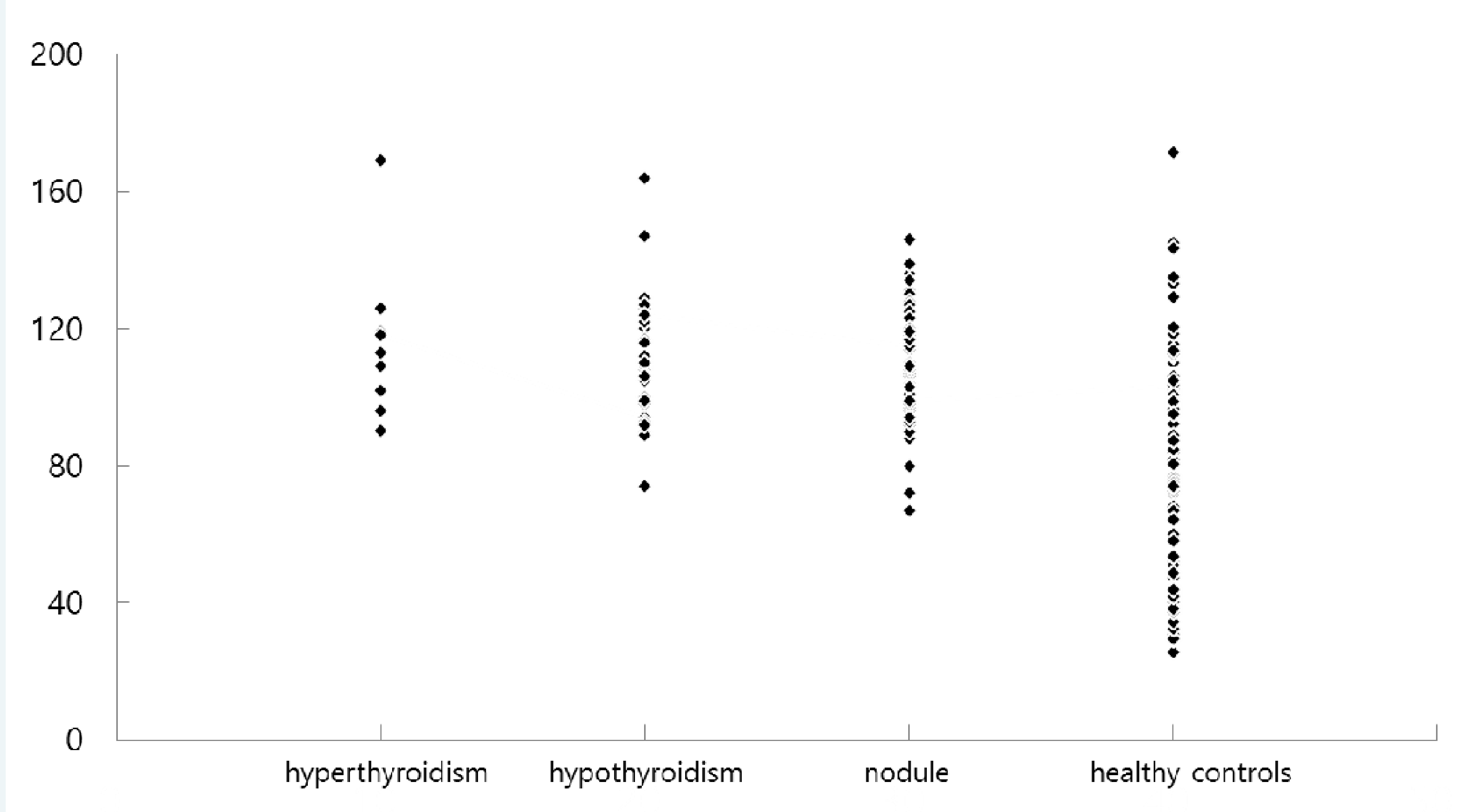

\section{Comparison of urine selenium concentrations of patients with various thyroid diseases to those of healthy subjects}

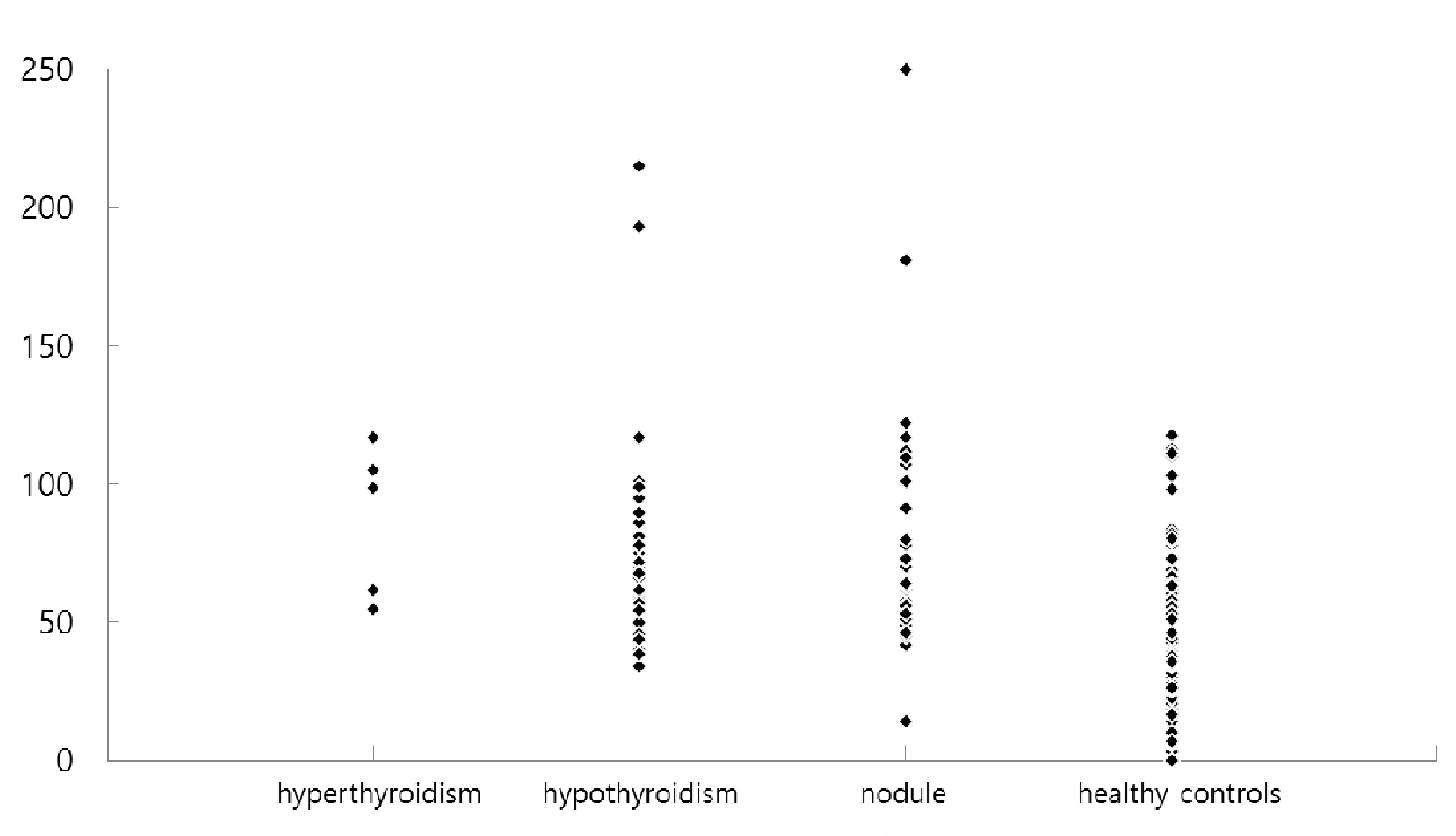

hyperthyroidism vs. controls; hypothyroidism vs. controls; nodule vs. controls, $\mathrm{p}<0.001$

When 97 patients were classified into 3 groups (hyperthyroidism, hypothyroidism, and benign thyroid nodule), and compared to 175 healthy subjects, serum and urine selenium concentrations of each 3 groups were not different from each other, but significantly higher than those of healthy subjects $(\mathrm{p}<0.001)$.

Serum selenium concentration was significantly correlated with urine selenium concentration after $\log$ transformation $(r=0.88, p=0.022)$ but not with UIC, T3, free T4 and TSH concentrations.

\section{CONCLUSION}

Selenium concentrations of the patients with thyroid disease were significantly higher than those of healthy subjects. Serum selenium concentration was significantly correlated with urine selenium concentration. 